\title{
SECTIONAL GEOMETRIC GENERA FOR AMPLE VECTOR BUNDLES
}

\author{
HiRONOBU ISHIHARA
}

\begin{abstract}
Two invariants, called the $c_{1}$-sectional geometric genus and the $\mathcal{O}(1)$-sectional geometric genus, are introduced for generalized polarized manifolds, i.e., pairs of a projective manifold and an ample vector bundle on it. They are generalizations of the $c_{1}$-sectional genus and the $\mathcal{O}(1)$-sectional genus introduced by T. Fujita for generalized polarized manifolds. They are also generalizations of the sectional geometric genus introduced by Y. Fukuma for polarized manifolds. We classify generalized polarized manifolds of the smallest $c_{1}$ or $\mathcal{O}(1)$-sectional geometric genus under the spannedness condition for ample vector bundles.
\end{abstract}

\section{Introduction}

For the classification of projective manifolds, ample line bundles are useful tools. A polarized manifold is the pair $(X, L)$ of a projective manifold $X$ and an ample line bundle $L$ on $X$. For the classification of polarized manifolds, the sectional genus $g(X, L)$ is an important invariant that is a natural generalization of the genus of curves. A generalized polarized manifold $(X, \mathscr{E})$ is the pair of a projective manifold $X$ and an ample vector bundle $\mathscr{E}$ on $X$. We note that generalized polarized manifolds $(X, \mathscr{E})$ are corresponding to the scrolls $\left(\boldsymbol{P}_{X}(\mathscr{E}), H(\mathscr{E})\right)$ that are basic polarized manifolds. (We denote by $H(\mathscr{E})$ the tautological line bundle $\mathcal{O}_{\boldsymbol{P}(\mathscr{E})}(1)$ on $\boldsymbol{P}_{X}(\mathscr{E})$.) T. Fujita $[\mathrm{F} 1]$ introduced the $c_{1}$-sectional genus $g(X$, det $\mathscr{E})$ and the $\mathcal{O}(1)$-sectional genus $g\left(\boldsymbol{P}_{X}(\mathscr{E}), H(\mathscr{E})\right)$ for the classification of generalized polarized manifolds. These sectional genera of generalized polarized manifolds are natural generalizations of the sectional genus of polarized manifolds (see also $[$ BiLL]).

On the other hand, A. Lanteri $[\mathrm{L}]$ introduced the geometric genus $p_{g}(X, \mathscr{E})$ for ample vector bundles $\mathscr{E}$ with regular sections, i.e., there exists a global section whose zero locus $Z$ is a submanifold of $X$ of the expected dimension. This genus is defined as the geometric genus $p_{g}(Z)$ of the submanifold $Z$. Recently, Y. Fukuma [Fk1] introduced the sectional geometric genus $g_{i}(X, L)$ for polarized

2000 Mathematics Subject Classification: Primary 14J60; Secondary 14C20, 14F05, 14J45.

Keywords and phrases: Vector bundles, sectional genus, geometric genus, polarized manifolds. Received March 31, 2003; revised July 23, 2003. 
manifolds $(X, L)$ and integers $0 \leq i \leq n$. This genus is a natural generalization of both the sectional genus and the geometric genus because $g_{1}(X, L)=g(X, L)$ and $g_{n}(X, L)=p_{g}(X)$. For every $0 \leq i \leq n, g_{i}(X, L)$ is a non-negative integer when $L$ is spanned (i.e. generated by global sections). In fact, $g_{i}(X, L) \geq h^{i}\left(\mathscr{O}_{X}\right)$ and we have classification results for the pairs $(X, L)$ of small $g_{2}(X, L)$ (w.r.t. $\left.h^{2}\left(\mathcal{O}_{X}\right)\right)$ in $[\mathrm{Fk} 1]$.

Motivated by the above papers [F1], [L] and [Fk1], we consider two invariants $g_{i}(X$, det $\mathscr{E})$ and $g_{i}\left(\boldsymbol{P}_{X}(\mathscr{E}), H(\mathscr{E})\right)$ for generalized polarized manifolds $(X, \mathscr{E})$. We call them the $c_{1}$-sectional geometric genus and the $\mathcal{O}(1)$-sectional geometric genus respectively. By definition, they are natural generalizations of both the $c_{1}$-sectional genus and the $\mathcal{O}(1)$-sectional genus, and also a generalization of the sectional geometric genus. When $\mathscr{E}$ is spanned, both $g_{i}(X$, det $\mathscr{E})$ and $g_{i}\left(\boldsymbol{P}_{X}(\mathscr{E}), H(\mathscr{E})\right)$ are non-negative integers and not less than $h^{i}\left(\mathcal{O}_{X}\right)$. We obtain classification results for the pairs $(X, \mathscr{E})$ with $g_{2}(X$, det $\mathscr{E})=h^{2}\left(\mathcal{O}_{X}\right)$ or $g_{2}\left(\boldsymbol{P}_{X}(\mathscr{E}), H(\mathscr{E})\right)=h^{2}\left(\mathcal{O}_{X}\right)$. As corollaries, we obtain the classification of pairs $(X, \mathscr{E})$ with $g_{2}(X, \operatorname{det} \mathscr{E})=0$ or $g_{2}\left(\boldsymbol{P}_{X}(\mathscr{E}), H(\mathscr{E})\right)=0$.

The contents of the present paper are as follows. In Section 1, we review the properties of the sectional geometric genus needed for the following sections. In Section 2 (resp. 3), we consider basic properties of the $c_{1}$ (resp. $\left.\mathcal{O}(1)\right)$-sectional geometric genus. In Appendix, we recall Lanteri's geometric genus of generalized polarized manifolds and consider its relation to the $c_{1}$-sectional geometric genus.

\section{Notation and Terminology}

We work over the complex number field. Varieties are always irreducible and reduced. Line bundles are identified with the linear equivalence classes of Cartier divisors. The tensor products of line bundles are denoted additively, while we use multiplicative notation for intersection products. Numerical equivalence is written as $\equiv$.

A vector bundle $\mathscr{E}$ is said to be ample if the tautological line bundle $H(\mathscr{E})$ on $\boldsymbol{P}_{X}(\mathscr{E})$ is ample. We say that $\mathscr{E}$ is spanned if $H(\mathscr{E})$ is spanned, i.e., generated by global sections. The restriction $\left.\mathscr{E}\right|_{W}$ of $\mathscr{E}$ to a variety $W$ is often denoted by $\mathscr{E} W$. We denote by $L^{\oplus r}$ the direct sum of $r$ copies of a line bundle $L$. For writing cohomology groups and Euler characteristic, we often omit the base spaces in case of no fear of confusion. We denote by $\rho(W)$ the Picard number of $W$.

A polarized manifold $(X, L)$ is said to be a Del Pezzo manifold if $K_{X}+$ $(n-1) L=\mathcal{O}_{X}$ for $n=\operatorname{dim} X$. We note that $(X, L)$ is said to be a scroll over a variety $W$ if $(X, L) \cong\left(\boldsymbol{P}_{W}(\mathscr{E}), H(\mathscr{E})\right)$ for some ample vector bundle $\mathscr{E}$ on $W$. We say that $(X, L)$ is a quadric fibration over $W$ if there exists a fibration $f: X \rightarrow W$ such that $\left(F, L_{F}\right) \cong\left(\boldsymbol{Q}^{m}, \mathcal{O}(1)\right)$ for a general fiber $F$ of $f$, where $\boldsymbol{Q}^{m}$ is a smooth hyperquadric in $\boldsymbol{P}^{m+1}$ and $m=\operatorname{dim} X-\operatorname{dim} W$. We call $(X, L)$ a Veronese fibration over a curve $C$ if there exists a fibration $f: X \rightarrow C$ such that $\left(F, L_{F}\right) \cong\left(\boldsymbol{P}^{2}, \mathcal{O}(2)\right)$ for every fiber $F$ of $f$. We say that $(X, L)$ is a simple blow- 
up of a polarized manifold $\left(X^{\prime}, L^{\prime}\right)$ if there exists a blowing-up $\pi: X \rightarrow X^{\prime}$ at a point $x^{\prime}$ and $L=\pi^{*} L^{\prime}-\pi^{-1}\left(x^{\prime}\right)$. A polarized manifold $\left(X^{\prime}, L^{\prime}\right)$ is called the reduction of $(X, L)$ if there exists a morphism $f: X \rightarrow X^{\prime}$ that is a composite of simple blow-ups and $\left(X^{\prime}, L^{\prime}\right)$ is not a simple blow-up of any polarized manifold.

\section{Sectional geometric genus}

Definition $1.1([\mathrm{Fk} 1,(2.1)])$. Let $X$ be a projective manifold of dimension $n$ and $L$ an ample line bundle on $X$. For every integer $0 \leq i \leq n$, the $i$-th sectional geometric genus $g_{i}(X, L)$ of the pair $(X, L)$ is defined by the formula

$$
g_{i}(X, L):=(-1)^{i}\left(\chi_{n-i}(X, L)-\chi\left(\mathcal{O}_{X}\right)\right)+\sum_{j=0}^{n-i}(-1)^{n-i-j} h^{n-j}\left(\mathcal{O}_{X}\right) .
$$

Here $\chi_{n-i}(X, L)$ is an integer that is uniquely determined by the Hilbert polynomial of $(X, L)$ :

$$
\chi(t L)=\sum_{j=0}^{n} \chi_{j}(X, L) t^{[j]} / j !
$$

where $t^{[0]}:=1$ and $t^{[j]}:=t(t+1) \cdots(t+j-1)$ for $1 \leq j \in \boldsymbol{Z}$.

Remark 1.1.1. We find that

$$
\begin{aligned}
& g_{n}(X, L)=h^{n}\left(\mathcal{O}_{X}\right)=p_{g}(X) \\
& g_{0}(X, L)=\chi_{n}(X, L)=L^{n}, \\
& g_{1}(X, L)=1-\chi_{n-1}(X, L)=1+\frac{1}{2}\left(K_{X}+(n-1) L\right) L^{n-1}
\end{aligned}
$$

by the Hirzebruch-Riemann-Roch theorem (see [F0,(I,2.1)]). Hence $g_{n}(X, L)$ is the geometric genus of $X, g_{0}(X, L)$ is the degree of $(X, L)$, and $g_{1}(X, L)$ is the sectional genus of $(X, L)$.

For a computation of the sectional geometric genus, we use the following formula.

Proposition $1.2([\mathrm{Fk} 1,(2.3)])$. Let $X$ be a projective manifold of dimension $n$ and $L$ an ample line bundle on $X$. For every integer $1 \leq i \leq n-1$, we have

$$
g_{i}(X, L)=\sum_{j=0}^{n-i-1}(-1)^{j}\left(\begin{array}{c}
n-i \\
j
\end{array}\right) h^{0}\left(K_{X}+(n-i-j) L\right)+\sum_{k=0}^{n-i}(-1)^{n-i-k} h^{n-k}\left(\mathcal{O}_{X}\right) .
$$

When $L$ is spanned, we get a smooth ladder of $(X, L)$ by Bertini's theorem. 
Proposition 1.3 (See, e.g., [F0,(I,4.1)]). Let $X$ be a projective manifold of dimension $n$ and $L$ an ample and spanned line bundle on $X$. Then there exists a sequence

$$
X=X_{n} \supset X_{n-1} \supset \cdots \supset X_{1}
$$

of submanifolds $X_{i}$ of $X$ such that $\operatorname{dim} X_{i}=i$ and $X_{i} \in\left|L_{X_{i+1}}\right|$ for every integer $1 \leq i \leq n-1$.

Remark 1.3.1. We note that the numbering of $\left\{X_{i}\right\}_{i=1}^{n}$ is different from that in $[\mathrm{Fk} 1]$. In the situation of (1.3), we find that

$$
g_{i}(X, L)=g_{i}\left(X_{n-1}, L_{X_{n-1}}\right)=\cdots=g_{i}\left(X_{i}, L_{X_{i}}\right)=p_{g}\left(X_{i}\right)
$$

for every integer $1 \leq i \leq n-1$ (cf. $[\mathrm{Fk} 1,(2.4)])$. This is the reason why $g_{i}(X, L)$ is called the $i$-th sectional geometric genus.

Existence of a ladder is essential for the following result.

Proposition 1.4 ([Fk1,(3.4.1)]). Let $X$ be a projective manifold of dimension $n$ and $L$ an ample and spanned line bundle on $X$. Then $g_{i}(X, L) \geq h^{i}\left(\mathcal{O}_{X}\right)$ for every integer $0 \leq i \leq n$. Moreover, when $n \geq 3, g_{2}(X, L)=h^{2}\left(\mathcal{O}_{X}\right)$ if and only if $(X, L)$ is one of the following:

(i) $\left(\boldsymbol{P}^{n}, \mathcal{O}(1)\right)$;

(ii) $\left(\boldsymbol{Q}^{n}, \mathcal{O}(1)\right)$;

(iii) a Del Pezzo manifold;

(iv) a scroll over a smooth curve;

(v) a quadric fibration over a smooth curve;

(vi) a scroll over a smooth surface;

(vii) the reduction $\left(X^{\prime}, L^{\prime}\right)$ of $(X, L)$ is either $\left(\boldsymbol{P}^{4}, \mathcal{O}(2)\right), \quad\left(\boldsymbol{P}^{3}, \mathcal{O}(3)\right)$, $\left(\boldsymbol{Q}^{3}, \mathcal{O}(2)\right)$, or a Veronese fibration over a smooth curve. (The case $(X, L)=\left(X^{\prime}, L^{\prime}\right)$ is included. $)$

Outline of Proof. We find that (cf. (1.3.1))

$$
\begin{aligned}
g_{i}(X, L) & =g_{i}\left(X_{i+1}, L_{X_{i+1}}\right) \\
& =h^{0}\left(K_{X_{i+1}}+L_{X_{i+1}}\right)-h^{0}\left(K_{X_{i+1}}\right)+h^{i}\left(\mathcal{O}_{X_{i+1}}\right) \\
& \geq h^{i}\left(\mathcal{O}_{X_{i+1}}\right) \\
& =h^{i}\left(\mathcal{O}_{X}\right) .
\end{aligned}
$$

If $g_{2}(X, L)=h^{2}\left(\mathcal{O}_{X}\right)$ and $n \geq 3$, then we get $h^{0}\left(K_{X_{3}}+L_{X_{3}}\right)=0$. It follows that $h^{0}\left(K_{X}+(n-2) L\right)=0$, hence $K_{X}+(n-2) L$ is not nef and $(X, L)$ is one of the above cases $(\mathrm{i}), \ldots$, (vii) by adjunction theory (see, e.g., [BS, Chap. 7]) or [F0,(II, $\S 11)])$. 
Remark 1.4.1. We see that $g_{2}(X, L)=0$ for all the cases in (1.4) except (vi); in Case (vi), $g_{2}(X, L)=0$ if and only if $p_{g}$ of the base surface is zero.

Remark 1.4.2. When $n \geq 2$, we have $g_{1}(X, L)=h^{1}\left(\mathcal{O}_{X}\right)$ if and only if $(X, L) \cong\left(\boldsymbol{P}^{n}, \mathcal{O}(1)\right),\left(\boldsymbol{P}^{2}, \mathcal{O}(2)\right),\left(\boldsymbol{Q}^{n}, \mathcal{O}(1)\right)$, or $(X, L)$ is a scroll over a smooth curve (see, e.g., [BS,(7.2.10)]). Moreover, it is known that $g_{1}(X, L) \geq 0$ for all polarized manifolds $(X, L)$, and if $g_{1}(X, L)=0$ and $n \geq 2$, then $(X, L) \cong$ $\left(\boldsymbol{P}^{n}, \mathcal{O}(1)\right),\left(\boldsymbol{P}^{2}, \mathcal{O}(2)\right), \quad\left(\boldsymbol{Q}^{n}, \mathcal{O}(1)\right)$, or $(X, L)$ is a scroll over $\boldsymbol{P}^{1}$ (see, e.g., $[\mathrm{F} 0,(\mathrm{II}, 12.1)])$. It is unknown, however, that $g_{i}(X, L) \geq 0$ for all polarized manifolds $(X, L)$ when $2 \leq i \leq n-1$.

Remark 1.4.3. Let $(X, L)$ be as in (1.4). Then $g_{i}(X, L)=h^{i}\left(\mathcal{O}_{X}\right)$ if and only if $H^{0}\left(K_{X}+(n-i) L\right)=0$ for every $i<n$ (see [Fk1,(3.3)]). Thus the classification of pairs $(X, L)$ with $g_{i}(X, L)=h^{i}\left(\mathcal{O}_{X}\right)$ becomes harder as $i$ increases. In [Fk2] we have a partial classification of pairs $(X, L)$ with $g_{3}(X, L)=0$ when $n \geq 5$ and $L$ is very ample.

\section{2. $c_{1}$-sectional geometric genus}

Definition 2.1. Let $X$ be a projective manifold of dimension $n$ and $\mathscr{E}$ an ample vector bundle of rank $r$ on $X$. The $i$-th $c_{1}$-sectional geometric genus of the pair $(X, \mathscr{E})$ is defined as $g_{i}(X$, det $\mathscr{E})$, where $g_{i}$ is the $i$-th sectional geometric genus (see (1.1)).

Remark 2.1.1. From (1.1.1) we see that $g_{n}(X$, det $\mathscr{E})=p_{g}(X), g_{0}(X$, det $\mathscr{E})$ $=c_{1}(\mathscr{E})^{n}$, and $g_{1}(X, \operatorname{det} \mathscr{E})$ is equal to the $c_{1}$-sectional genus of $(X, \mathscr{E})$ (see [F1]). When $r=1$, the $c_{1}$-sectional geometric genus is nothing but the sectional geometric genus.

Remark 2.1.2. When $\mathscr{E}$ is spanned, from (1.4) we see that $g_{i}(X, \operatorname{det} \mathscr{E}) \geq$ $h^{i}\left(\mathcal{O}_{X}\right)$ for every $0 \leq i \leq n$.

For $i=1$ we have the following classification result.

Proposition 2.2. Let $X$ be a projective manifold of dimension $n \geq 2$ and $\mathscr{E}$ an ample and spanned vector bundle of rank $r \geq 2$ on $X$. Then $g_{1}(X$, det $\mathscr{E}) \geq$ $h^{1}\left(\mathcal{O}_{X}\right)$, and equality holds if and only if $(X, \mathscr{E}) \cong\left(\boldsymbol{P}^{2}, \mathcal{O}(1)^{\oplus 2}\right)$. Moreover, $g_{1}(X$, det $\mathscr{E})=h^{1}\left(\mathcal{O}_{X}\right)+1$ if and only if $(X, \mathscr{E})$ is one of the following:
(i) $\left(\boldsymbol{P}^{3}, \mathcal{O}(1)^{\oplus 2}\right)$;
(ii) $\left(\boldsymbol{P}^{2}, \mathcal{O}(1)^{\oplus 3}\right)$;
(iii) $\left(\boldsymbol{P}^{2}, \mathcal{O}(1) \oplus \mathcal{O}(2)\right)$;
(iv) $\left(\boldsymbol{P}^{2}, \mathscr{T}_{\boldsymbol{P}^{2}}\right)$, where $\mathscr{T}_{\boldsymbol{P}^{2}}$ is the tangent bundle of $\boldsymbol{P}^{2}$;
(v) $\left(\boldsymbol{P}^{1} \times \boldsymbol{P}^{1}, \mathcal{O}(1,1)^{\oplus 2}\right)$. 
Proof. The inequality $g_{1}(X$, det $\mathscr{E}) \geq h^{1}\left(\mathcal{O}_{X}\right)$ follows by (2.1.2). Suppose that $g_{1}(X$, det $\mathscr{E})=h^{1}\left(\mathcal{O}_{X}\right)$. We note that $(\operatorname{det} \mathscr{E}) C \geq r$ for any rational curve $C$ in $X$ since $\mathscr{E}$ is ample. Then we see that $(X$, det $\mathscr{E}) \cong\left(\boldsymbol{P}^{2}, \mathcal{O}(2)\right)$ from (1.4.2). It follows that $\left.\mathscr{E}\right|_{l} \cong \mathcal{O}_{\boldsymbol{P}^{1}}(1)^{\oplus 2}$ for every line $l$ in $\boldsymbol{P}^{2}$, hence $\mathscr{E}$ is a uniform vector bundle and $\mathscr{E} \cong \mathcal{O}_{\boldsymbol{P}^{2}}(1)^{\oplus 2}$ by, e.g., [OSS,(I,3.2.1)]. Conversely, if $(X, \mathscr{E}) \cong\left(\boldsymbol{P}^{2}\right.$, $\left.\mathcal{O}(1)^{\oplus 2}\right)$, then $g_{1}(X$, det $\mathscr{E})=h^{1}\left(\mathcal{O}_{X}\right)=0$.

Suppose that $g_{1}(X$, det $\mathscr{E})=h^{1}\left(\mathcal{O}_{X}\right)+1$. Then, by [FkI, (3.2)], $(X$, det $\mathscr{E})$ is a Del Pezzo manifold unless

(*) $n=2, X \cong \boldsymbol{P}_{C}(\mathscr{F})$ and $\operatorname{det} \mathscr{E} \equiv 2 H(\mathscr{F})$ for an ample vector bundle $\mathscr{F}$ of rank 2 on an elliptic curve $C$ with $c_{1}(\mathscr{F})=1$.

If $(X, \operatorname{det} \mathscr{E})$ is the case $(*)$, then $g_{1}(X, \operatorname{det} \mathscr{E})=2$ and we see that $\mathscr{E}$ is not spanned because of $[\operatorname{BiLL},(1.3)]$. Thus $(X$, det $\mathscr{E})$ is a Del Pezzo manifold. Then $g_{1}(X, \operatorname{det} \mathscr{E})=1$ and $(X, \mathscr{E})$ is one of the above cases $(\mathrm{i}), \ldots,(\mathrm{v})$ by $[\mathrm{F} 1,(1.5)]$. Conversely, $h^{1}\left(\mathcal{O}_{X}\right)=0$ and $g_{1}(X$, det $\mathscr{E})=1$ for all the cases (i) $, \ldots,(\mathrm{v})$.

Remark 2.2.1. In fact, by $[\mathrm{F} 1,(1.4) \&(1.5)], g_{1}(X$, det $\mathscr{E}) \geq 0$ for all generalized polarized manifolds $(X, \mathscr{E})$, and equality holds if and only if $(X, \mathscr{E}) \cong$ $\left(\boldsymbol{P}^{2}, \mathcal{O}(1)^{\oplus 2}\right)$; moreover, $g_{1}(X$, det $\mathscr{E})=1$ if and only if $(X, \mathscr{E})$ is one of the cases (i), .., (v) of (2.2).

For $i=2$ we obtain the following.

THEOREM 2.3. Let $X$ be a projective manifold of dimension $n \geq 3$ and $\mathscr{E}$ an ample and spanned vector bundle of rank $r \geq 2$ on $X$. Then $g_{2}(X$, det $\mathscr{E}) \geq$ $h^{2}\left(\mathcal{O}_{X}\right)$, and equality holds if and only if $(X, \mathscr{E})$ is one of the following:

(i) $\left(\boldsymbol{P}^{4}, \mathcal{O}(1)^{\oplus 2}\right)$;

(ii) $\left(\boldsymbol{P}^{3}, \mathcal{O}(1)^{\oplus 3}\right)$;

(iii) $\left(\boldsymbol{P}^{3}, \mathcal{O}(1)^{\oplus 2}\right)$;

(iv) $\left(\boldsymbol{P}^{3}, \mathcal{O}(1) \oplus \mathcal{O}(2)\right)$;

(v) $\left(\boldsymbol{Q}^{3}, \mathcal{O}(1)^{\oplus 2}\right)$;

(vi) $\left(\boldsymbol{P}_{W}(\mathscr{F}), H(\mathscr{F}) \otimes \varphi^{*} \mathscr{G}\right)$, where $\mathscr{F}$ and $\mathscr{G}$ are vector bundles on a smooth curve $W$ such that $n=\operatorname{rank} \mathscr{F}=3, r=\operatorname{rank} \mathscr{G}=2$ and $\varphi: \boldsymbol{P}_{W}(\mathscr{F}) \rightarrow W$ is the bundle projection.

Proof. The inequality $g_{2}(X$, det $\mathscr{E}) \geq h^{2}\left(\mathcal{O}_{X}\right)$ follows by (2.1.2). Suppose that $g_{2}(X, \operatorname{det} \mathscr{E})=h^{2}\left(\mathcal{O}_{X}\right)$. Then the pair $(X$, det $\mathscr{E})$ is one of the cases of (1.4). Since $\mathscr{E}$ is ample, we see that $(\operatorname{det} \mathscr{E}) C \geq 2$ for any rational curve $C$ in $X$. Hence $(X$, det $\mathscr{E})$ is the reduction of itself and $(X$, det $\mathscr{E})$ is one of the following:

(a) a Del Pezzo manifold;

(b) $\left(\boldsymbol{P}^{3}, \mathcal{O}(3)\right)$;

(c) $\left(\boldsymbol{P}^{4}, \mathcal{O}(2)\right)$;

(d) $\left(Q^{3}, \mathcal{O}(2)\right)$;

(e) a Veronese fibration over a smooth curve.

In Case $\left(\right.$ a), we have $g_{1}(X, \operatorname{det} \mathscr{E})=1$, hence $(X, \mathscr{E}) \cong\left(\boldsymbol{P}^{3}, \mathcal{O}(1)^{\oplus 2}\right)$ by $(2.2)$. 
In Case (b), we see that $r=2$ or 3 since $(\operatorname{det} \mathscr{E}) l=3$ for every line $l$ in $\boldsymbol{P}^{3}$. Moreover, if $r=2$, we have $\left.\mathscr{E}\right|_{l} \cong \mathcal{O}_{\boldsymbol{P}^{1}}(1) \oplus \mathcal{O}_{\boldsymbol{P}^{1}}(2)$; if $r=3$, we have $\left.\mathscr{E}\right|_{l} \cong \mathcal{O}_{\boldsymbol{P}^{1}}(1)^{\oplus 3}$. Hence we get $\mathscr{E} \cong \mathcal{O}_{\boldsymbol{P}^{3}}(1) \oplus \mathcal{O}_{\boldsymbol{P}^{3}}(2)$ or $\mathcal{O}_{\boldsymbol{P}^{3}}(1)^{\oplus 3}$ by, e.g., [OSS,(I,3.2.1)\&(I,3.2.3)].

In Case (c), similarly as above, we have $\left.\mathscr{E}\right|_{l} \cong \mathcal{O}_{\boldsymbol{P}^{1}}(1)^{\oplus 2}$ for every line $l$ in $\boldsymbol{P}^{4}$. Hence $\mathscr{E} \cong \mathcal{O}_{\boldsymbol{P}^{4}}(1)^{\oplus 2}$.

In Case $(\mathrm{d})$, we have $\left.\mathscr{E}\right|_{l} \cong \mathcal{O}_{\boldsymbol{P}^{1}}(1)^{\oplus 2}$ for every line $l$ on $\boldsymbol{Q}^{3}$ in $\boldsymbol{P}^{4}$. Hence $\mathscr{E} \cong \mathcal{O}_{Q^{3}}(1)^{\oplus 2}$ by the argument in [YZ, p. 679,(4.3)].

In Case (e), let $\varphi: X \rightarrow W$ be a Veronese fibration over a smooth curve such that $\operatorname{det} \mathscr{E}_{F}=\mathcal{O}_{\boldsymbol{P}^{2}}(2)$ for every fiber $F \cong \boldsymbol{P}^{2}$ of $\varphi$. Then $\mathscr{E}_{F} \cong \mathcal{O}_{\boldsymbol{P}^{2}}(1)^{\oplus 2}$ and $h^{0}\left(F,\left(K_{X}+2 \operatorname{det} \mathscr{E}\right)_{F}\right)=3$ for every fiber $F$ of $\varphi$. Hence $\mathscr{F}:=\varphi_{*} \mathcal{O}_{X}\left(K_{X}+\right.$ $2 \operatorname{det} \mathscr{E})$ is a locally free sheaf of rank 3 and it follows that $X \cong \boldsymbol{P}_{W}(\mathscr{F})$. Then $h^{0}\left(F,(\mathscr{E} \otimes(-H(\mathscr{F})))_{F}\right)=2$ for every fiber $F$ of $\varphi$. Hence $\mathscr{G}:=\varphi_{*}(\mathscr{E} \otimes$ $(-H(\mathscr{F})))$ is a locally free sheaf of rank 2 and it follows that $\varphi^{*} \mathscr{G} \cong \mathscr{E} \otimes$ $(-H(\mathscr{F}))$.

Thus we obtain the cases (i),.., (vi) of our theorem. Conversely, by (1.2), we find that $g_{2}(X, \operatorname{det} \mathscr{E})=h^{2}\left(\mathcal{O}_{X}\right)=0$ for all the cases $(\mathrm{i}), \ldots,(\mathrm{vi})$.

Remark 2.3.1. The case (vi) of (2.3) exists for an arbitrary smooth curve $W$. Indeed, let $\mathscr{F}$ (resp. $\mathscr{G}$ ) be an ample and spanned vector bundle of rank 3 (resp. 2) on $W$. We set $(X, \mathscr{E}):=\left(\boldsymbol{P}_{W}(\mathscr{F}), H(\mathscr{F}) \otimes \varphi^{*} \mathscr{G}\right)$, where $\varphi: \boldsymbol{P}_{W}(\mathscr{F}) \rightarrow W$ is the bundle projection. Then $\mathscr{E}$ is ample and spanned, and by (1.2) we find that

$$
\begin{aligned}
g_{2}(X, \operatorname{det} \mathscr{E}) & =h^{0}\left(K_{X}+\operatorname{det} \mathscr{E}\right)-h^{3}\left(\mathcal{O}_{X}\right)+h^{2}\left(\mathcal{O}_{X}\right) \\
& =h^{0}\left(-H(\mathscr{F})+\varphi^{*}\left(K_{W}+\operatorname{det} \mathscr{F}+\operatorname{det} \mathscr{G}\right)\right) \\
& =0 .
\end{aligned}
$$

COROllary 2.4. Let $X$ be a projective manifold of dimension $n \geq 3$ and $\mathscr{E}$ an ample and spanned vector bundle of rank $r \geq 2$ on $X$. Then $g_{2}(X$, det $\mathscr{E}) \geq 0$, and equality holds if and only if $(X, \mathscr{E})$ is one of the cases of (2.3).

Remark 2.4.1. The classification of pairs $(X, \mathscr{E})$ with $g_{i}(X$, det $\mathscr{E})=0$ and $i \geq 3$ is yet to be studied (cf. (1.4.3)).

\section{3. $\mathcal{O}(1)$-sectional geometric genus}

DeFINITION 3.1. Let $X$ be a projective manifold of dimension $n$ and $\mathscr{E}$ an ample vector bundle of rank $r$ on $X$. The $i$-th $\mathcal{O}(1)$-sectional geometric genus of the pair $(X, \mathscr{E})$ is defined as $g_{i}\left(\boldsymbol{P}_{X}(\mathscr{E}), H(\mathscr{E})\right)$, where $g_{i}$ is the $i$-th sectional geometric genus (see (1.1)).

Remark 3.1.1. From (1.1.1) we see that $g_{0}\left(\boldsymbol{P}_{X}(\mathscr{E}), H(\mathscr{E})\right)=H(\mathscr{E})^{n+r-1}=$ $s_{n}(\mathscr{E})$ and $g_{1}\left(\boldsymbol{P}_{X}(\mathscr{E}), H(\mathscr{E})\right)$ is equal to the $\mathcal{O}(1)$-sectional genus of $(X, \mathscr{E})$ (see 
$[\mathrm{F} 1])$. When $r=1$, the $\mathcal{O}(1)$-sectional geometric genus is nothing but the sectional geometric genus.

Remark 3.1.2. By (1.2) and the Kodaira vanishing theorem, we get

$$
g_{i}\left(\boldsymbol{P}_{X}(\mathscr{E}), H(\mathscr{E})\right)= \begin{cases}0 & \text { for } i \geq n+1, \\ p_{g}(X) & \text { for } i=n, \\ g_{n-1}(X, \operatorname{det} \mathscr{E}) & \text { for } i=n-1\end{cases}
$$

$($ See $[F k 1,(2.10 .8)]$.

Remark 3.1.3. When $\mathscr{E}$ is spanned, from (1.4) we see that $g_{i}\left(\boldsymbol{P}_{X}(\mathscr{E}), H(\mathscr{E})\right) \geq$ $h^{i}\left(\mathcal{O}_{\boldsymbol{P}(\mathscr{E})}\right)=h^{i}\left(\mathcal{O}_{X}\right)$ for every $0 \leq i \leq n+r-1$.

For $i=1$ we have the following classification result.

Proposition 3.2. Let $X$ be a projective manifold of dimension $n \geq 2$ and $\mathscr{E}$ an ample and spanned vector bundle of rank $r \geq 2$ on $X$. Then $g_{1}\left(\boldsymbol{P}_{X}(\mathscr{E}), H(\mathscr{E})\right) \geq$ $h^{1}\left(\mathcal{O}_{X}\right)$, and equality holds if and only if $(X, \mathscr{E}) \cong\left(\boldsymbol{P}^{n}, \mathcal{O}(1)^{\oplus 2}\right)$. Moreover, $g_{1}\left(\boldsymbol{P}_{X}(\mathscr{E}), H(\mathscr{E})\right)=h^{1}\left(\mathcal{O}_{X}\right)+1$ if and only if $(X, \mathscr{E})$ is one of the following:

(i) $\left(\boldsymbol{P}^{2}, \mathcal{O}(1)^{\oplus 3}\right)$;

(ii) $\left(\boldsymbol{P}^{2}, \mathcal{O}(1) \oplus \mathcal{O}(2)\right)$;

(iii) $\left(\boldsymbol{P}^{2}, \mathscr{T}_{\boldsymbol{P}^{2}}\right)$;

(iv) $\left(\boldsymbol{P}^{1} \times \boldsymbol{P}^{1}, \mathcal{O}(1,1)^{\oplus 2}\right)$.

Proof. The inequality $g_{1}\left(\boldsymbol{P}_{X}(\mathscr{E}), H(\mathscr{E})\right) \geq h^{1}\left(\mathcal{O}_{X}\right)$ follows by (3.1.3). Suppose that $g_{1}\left(\boldsymbol{P}_{X}(\mathscr{E}), H(\mathscr{E})\right)=h^{1}\left(\mathcal{O}_{X}\right)$ and let $p: \boldsymbol{P}_{X}(\mathscr{E}) \rightarrow X$ be the bundle projection. From (1.4.2) we see that $\left(\boldsymbol{P}_{X}(\mathscr{E}), H(\mathscr{E})\right)$ is a scroll over a smooth curve; let $f: \boldsymbol{P}_{X}(\mathscr{E}) \rightarrow Y$ be its scroll map. Since $p$ and $f$ are contraction morphisms of different extremal rays, any curve contained in a fiber of $p$ (resp. $f$ ) does not be contracted by $f$ (resp. $p$ ). Then, for a fiber $Z \cong \boldsymbol{P}^{r-1}$ of $p$, we see that $\left.f\right|_{Z}: \boldsymbol{P}^{r-1} \rightarrow Y$ is a finite morphism. Hence $r=2$ and $Y \cong \boldsymbol{P}^{1}$. On the other hand, for a fiber $F \cong \boldsymbol{P}^{n}$ of $f$, we see that $\left.p\right|_{F}: \boldsymbol{P}^{n} \rightarrow X$ is finite surjective. Hence $X \cong \boldsymbol{P}^{n}$ by $[\mathrm{Lz},(4.1)]$. Thus $\boldsymbol{P}_{X}(\mathscr{E})$ has two projective space bundle structures over projective spaces. Then, by $\left[\mathrm{Sa}\right.$, Theorem A], we get $\boldsymbol{P}_{X}(\mathscr{E}) \cong$

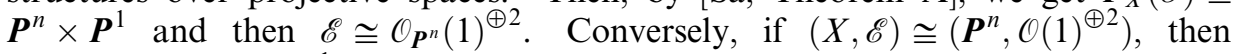
$g_{1}\left(\boldsymbol{P}_{X}(\mathscr{E}), H(\mathscr{E})\right)=h^{1}\left(\mathcal{O}_{X}\right)=0$.

Suppose that $g_{1}\left(\boldsymbol{P}_{X}(\mathscr{E}), H(\mathscr{E})\right)=h^{1}\left(\mathcal{O}_{X}\right)+1$. Then, by [FkI, (3.2)], $\left(\boldsymbol{P}_{X}(\mathscr{E})\right.$, $H(\mathscr{E}))$ is a Del Pezzo manifold since $\operatorname{dim} \boldsymbol{P}_{X}(\mathscr{E}) \geq 3$. It follows that $g_{1}\left(\boldsymbol{P}_{X}(\mathscr{E})\right.$, $H(\mathscr{E}))=1$, hence $(X, \mathscr{E})$ is one of the cases (i), ., (iv) of our theorem by $[\mathrm{F} 1,(3.3)]$. Conversely, $h^{1}\left(\mathcal{O}_{X}\right)=0$ and $g_{1}\left(\boldsymbol{P}_{X}(\mathscr{E}), H(\mathscr{E})\right)=1$ for all the cases (i),$\ldots$, (iv).

Remark 3.2.1. In fact, by $[\mathrm{F} 1,(3.2) \&(3.3)], g_{1}\left(\boldsymbol{P}_{X}(\mathscr{E}), H(\mathscr{E})\right) \geq 0$ for all generalized polarized manifolds $(X, \mathscr{E})$, and equality holds if and only if either 
$X \cong \boldsymbol{P}^{1}$ or $(X, \mathscr{E}) \cong\left(\boldsymbol{P}^{n}, \mathcal{O}(1)^{\oplus 2}\right)$; moreover, $g_{1}\left(\boldsymbol{P}_{X}(\mathscr{E}), H(\mathscr{E})\right)=1$ if and only if either $X$ is an elliptic curve or $(X, \mathscr{E})$ is one of the cases (i), .,,(iv) of (3.2).

For $i=2$ we obtain the following.

THEOREM 3.3. Let $X$ be a projective manifold of dimension $n \geq 3$ and $\mathscr{E}$ an ample and spanned vector bundle of rank $r \geq 2$ on $X$. Then $g_{2}\left(\boldsymbol{P}_{X}(\mathscr{E}), H(\mathscr{E})\right) \geq$ $h^{2}\left(\mathcal{O}_{X}\right)$, and equality holds if and only if $(X, \mathscr{E})$ is one of the following:

(i) $\left(\boldsymbol{P}^{n}, \mathcal{O}(1)^{\oplus 3}\right)$;

(ii) $\left(\boldsymbol{P}^{n}, \mathcal{O}(1)^{\oplus 2}\right)$;

(iii) $\left(\boldsymbol{P}^{3}, \mathcal{O}(1) \oplus \mathcal{O}(2)\right)$;

(iv) $\left(\boldsymbol{Q}^{n}, \mathcal{O}(1)^{\oplus 2}\right)$;

(v) $\left(\boldsymbol{P}_{W}(\mathscr{F}), H(\mathscr{F}) \otimes \varphi^{*} \mathscr{G}\right)$, where $\mathscr{F}$ and $\mathscr{G}$ are vector bundles on a smooth curve $W$ such that $n=\operatorname{rank} \mathscr{F} \geq 3, r=\operatorname{rank} \mathscr{G}=2$ and $\varphi: \boldsymbol{P}_{W}(\mathscr{F}) \rightarrow W$ is the bundle projection;

Proof. The inequality $g_{2}\left(\boldsymbol{P}_{X}(\mathscr{E}), H(\mathscr{E})\right) \geq h^{2}\left(\mathcal{O}_{X}\right)$ follows by (3.1.3). Suppose that $g_{2}\left(\boldsymbol{P}_{X}(\mathscr{E}), H(\mathscr{E})\right)=h^{2}\left(\mathcal{O}_{X}\right)$. Let $(P, H):=\left(\boldsymbol{P}_{X}(\mathscr{E}), H(\mathscr{E})\right), N:=\operatorname{dim} P$ $=n+r-1$ and $p: P \rightarrow X$ be the bundle projection. From (1.4) we see that $K_{P}+(N-2) H$ is not nef. Then there exists an extremal ray $R$ of $P$ such that $\left(K_{P}+(N-2) H\right) R<0$. Let $f: P \rightarrow Y$ be the contraction morphism of $R$. Since $K_{P}+(N-2) H=(n-3) H+p^{*}\left(K_{X}+\operatorname{det} \mathscr{E}\right)$, we see that $f \neq p$, $K_{X}+\operatorname{det} \mathscr{E}$ is not nef and there exists an extremal ray $R^{\prime}$ of $X$ such that $\left(K_{X}+\operatorname{det} \mathscr{E}\right) R^{\prime}<0$. Let $\varphi: X \rightarrow W$ be the contraction morphism of $R^{\prime}$. We can take $R^{\prime}$ that corresponds to $R$ and makes the following commutative diagram:

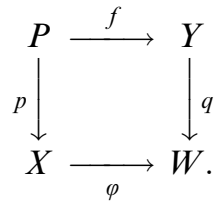

Since $\operatorname{dim} P \geq 4$ and $\rho(P)=\rho(X)+1 \geq 2$, from (1.4) we see that $f: P \rightarrow Y$ is one of the following:

(a) $(P, H)$ is a Del Pezzo manifold;

(b) a scroll over a smooth curve;

(c) a quadric fibration over a smooth curve;

(d) a scroll over a smooth surface;

(e) the reduction of $(P, H)$ is $\left(\boldsymbol{P}^{4}, \mathcal{O}(2)\right)$.

In Case (a), we have $g_{1}(P, H)=1$, hence the condition $n \geq 3$ is impossible because of (3.2).

In Case (b), $Y$ is a smooth curve. Since $p$ and $f$ are contraction morphisms of different extremal rays, any curve contained in a fiber of $p$ (resp. $f$ ) does not be contracted by $f$ (resp. $p$ ). Hence $Y=f(C)$ for a rational curve $C$ in a fiber 
of $p$. It follows that $Y \cong \boldsymbol{P}^{1}$ and then $g_{1}(P, H)=0$. Hence we get $(X, \mathscr{E}) \cong$ $\left(\boldsymbol{P}^{n}, \mathcal{O}(1)^{\oplus 2}\right)$ by $(3.2)$.

In Case (c), $Y$ is a smooth curve. From the argument above, for a fiber $Z \cong \boldsymbol{P}^{r-1}$ of $p$, we see that $\left.f\right|_{Z}: \boldsymbol{P}^{r-1} \rightarrow Y$ is a finite morphism. Hence $r=2$ and $Y \cong \boldsymbol{P}^{1}$. Then, for a general fiber $F \cong \boldsymbol{Q}^{n}$ of $f$, we see that $\left.p\right|_{F}: \boldsymbol{Q}^{n} \rightarrow X$ is finite surjective. Hence, by [PS, Prop. 8], $\left.p\right|_{F}$ is an isomorphism unless $X \cong \boldsymbol{P}^{n}$. We show that the case $X \cong \boldsymbol{P}^{n}$ does not occur. If $X \cong \boldsymbol{P}^{n}$, we take general hyperplane sections of $X$ and get a smooth surface $S \cong \boldsymbol{P}^{2}$. We consider a 3-dimensional scroll $\left(P^{\prime}, H^{\prime}\right):=\left(P_{S}\left(\mathscr{E}_{S}\right), H\left(\mathscr{E}_{S}\right)\right)$ that is a quadric fibration over $\boldsymbol{P}^{1}$ via $\left.f\right|_{p^{-1}(S)}=\left.f\right|_{P^{\prime}}$. Let $F^{\prime} \cong \boldsymbol{Q}^{2}$ be a general fiber of $\left.f\right|_{P^{\prime}}$. We have $F^{\prime} \in\left|a H^{\prime}-\left(\left.p\right|_{P^{\prime}}\right)^{*} \mathcal{O}_{P^{2}}(b)\right|$ and $\operatorname{det} \mathscr{E}_{S}=\mathcal{O}_{P^{2}}(e)$ for some integers $a, b$ and $e$. Then we find that

$$
\begin{aligned}
& 0=\left(F^{\prime}\right)^{3}=a^{3}\left(e^{2}-c_{2}\left(\mathscr{E}_{S}\right)\right)-3 a^{2} b e+3 a b^{2}, \\
& 0=H^{\prime} \cdot\left(F^{\prime}\right)^{2}=a^{2}\left(e^{2}-c_{2}\left(\mathscr{E}_{S}\right)\right)-2 a b e+b^{2} .
\end{aligned}
$$

By combining these equalities, we get $a e=2 b$. Moreover, we have

$$
\mathcal{O}_{F^{\prime}}=\left.\mathcal{O}_{P^{\prime}}\left(F^{\prime}\right)\right|_{F^{\prime}}=\left.a H^{\prime}\right|_{F^{\prime}}-\left(\left.p\right|_{F^{\prime}}\right)^{*} \mathcal{O}_{\boldsymbol{P}^{2}}(b)=\mathcal{O}_{\boldsymbol{Q}^{2}}(a-b c)
$$

for some positive integer $c$. It follows that $a=b c$ and then $c e=2$, hence $c=1$ and $e=2$. Then we get $\mathscr{E}_{S} \cong \mathcal{O}_{\boldsymbol{P}^{2}}(1)^{\oplus 2}$, which is a contradiction since $\left.f\right|_{p^{-1}(S)}$ is a quadric fibration. Thus we infer that $\left.p\right|_{F}$ is an isomorphism and $X \cong \boldsymbol{Q}^{n}$. By restricting $K_{P}=-2 H+p^{*}\left(K_{X}+\operatorname{det} \mathscr{E}\right)$ to $F$, we get det $\mathscr{E}=\mathcal{O}_{Q^{n}}(2)$. Hence $\mathscr{E} \cong \mathcal{O}_{Q^{n}}(1)^{\oplus 2}$ by the argument in [YZ, p. 679,(4.3)].

In Case $(\mathrm{d}), Y$ is a smooth surface and $(P, H) \cong\left(\boldsymbol{P}_{Y}\left(\mathscr{E}^{\prime}\right), H\left(\mathscr{E}^{\prime}\right)\right)$ for an ample vector bundle $\mathscr{E}^{\prime}$ on $Y$. Similarly as in Cases (b) and (c), for a fiber $Z \cong \boldsymbol{P}^{r-1}$ of $p$, we see that $\left.f\right|_{Z}: \boldsymbol{P}^{r-1} \rightarrow Y$ is finite. Hence $r=2$ or 3 . When $r=3,\left.f\right|_{Z}$ is surjective; also $\left.p\right|_{F}: F \rightarrow X$ is surjective for a fiber $F \cong \boldsymbol{P}^{n}$ of $f$. It follows that $Y \cong \boldsymbol{P}^{2}$ and $X \cong \boldsymbol{P}^{n}$ by [Lz,(4.1)]. Hence, by [Sa, Theorem A], we get $P \cong \boldsymbol{P}^{n} \times \boldsymbol{P}^{2}$ and then $\mathscr{E} \cong \mathcal{O}_{\boldsymbol{P}^{n}}(1)^{\oplus 3}$.

When $r=2$, we have $\operatorname{dim} p(F)=n-1$, hence $\operatorname{dim} W \leq 1$. We show that the case $\operatorname{dim} W=0$ does not occur. If $\operatorname{dim} W=0$, then we get $\rho(Y)=\rho(X)$ $=1$. It follows that $Y \cong \boldsymbol{P}^{2}$ since $Y$ is covered by rational curves $\left\{C_{x}:=\right.$ $\left.f\left(p^{-1}(x)\right) \mid x \in X\right\}$. By an argument similar to [YZ, p. 683, Lemma 10], we infer that $\left.f\right|_{p^{-1}(x)}: \boldsymbol{P}^{1} \rightarrow C_{x}$ is birational for every $x \in X$. Then we have

$$
\left(K_{Y}+\operatorname{det} \mathscr{E}^{\prime}\right) C_{x}=f^{*}\left(K_{Y}+\operatorname{det} \mathscr{E}^{\prime}\right) \cdot p^{-1}(x)=\left(K_{P}+n H\right) \cdot p^{-1}(x)=n-2,
$$

hence $(d-3) c=n-2$, where $c:=\operatorname{deg} C_{x}$ and $d:=c_{1}\left(\mathscr{E}^{\prime}\right)$. Since $\mathscr{E}^{\prime}$ is ample, we have $d \geq \operatorname{rank} \mathscr{E}^{\prime}=n$. If $c \geq 2$, then $d=n=4$ and $\left.\mathscr{E}^{\prime}\right|_{l} \cong \mathcal{O}_{\boldsymbol{P}^{1}}(1)^{\oplus 4}$ for every line $l \cong \boldsymbol{P}^{1}$ in $Y \cong \boldsymbol{P}^{2}$. It follows that $\mathscr{E}^{\prime} \cong \mathcal{O}_{\boldsymbol{P}^{2}}(1)^{\oplus 4}$ and $P \cong \boldsymbol{P}^{2} \times \boldsymbol{P}^{3}$, which is a contradiction since $p: P \rightarrow X$ is a $\boldsymbol{P}^{1}$-bundle. Thus $c=1$ and $d=n+1$. Then we have $\left.\mathscr{E}^{\prime}\right|_{l} \cong \mathcal{O}_{\boldsymbol{P}^{1}}(1)^{\oplus n-1} \oplus \mathcal{O}_{\boldsymbol{P}^{1}}(2)$, hence $\mathscr{E}^{\prime} \cong \mathcal{O}_{\boldsymbol{P}^{2}}(1)^{\oplus n-1} \oplus \mathcal{O}_{\boldsymbol{P}^{2}}(2)$. A natural surjection $\mathscr{E}^{\prime} \rightarrow \mathcal{O}_{\boldsymbol{P}^{2}}(1)^{\oplus n-1}$ determines a divisor $E:=\boldsymbol{P}\left(\mathcal{O}_{\boldsymbol{P}^{2}}(1)^{\oplus n-1}\right) \in$ 
$\left|H-f^{*} \mathcal{O}_{\boldsymbol{P}^{2}}(2)\right|$ on $P$. Then we get $E \cdot p^{-1}(x)=-1$, hence $p^{-1}(x) \subset E$ for all $x \in X$, which is a contradiction too.

Thus we have $\operatorname{dim} W=1$ and a general fiber $D$ of $\varphi$ is $p(F)$. Since $F \cong \boldsymbol{P}^{n-1}$, we see that $D \cong \boldsymbol{P}^{n-1}$ and $\varphi$ is a $\boldsymbol{P}^{n-1}$-fibration. We consider an $n$ dimensional scroll $\left(\boldsymbol{P}_{D}\left(\mathscr{E}_{D}\right), H\left(\mathscr{E}_{D}\right)\right)$ that is also a scroll over $f\left(p^{-1}(D)\right) \cong \boldsymbol{P}^{1}$ via $\left.f\right|_{p^{-1}(D)}$. By $[\mathrm{Sa}$, Theorem $\mathrm{A}]$, we get $\boldsymbol{P}_{D}\left(\mathscr{E}_{D}\right) \cong \boldsymbol{P}^{n-1} \times \boldsymbol{P}^{1}$ and then $\mathscr{E}_{D} \cong$ $\mathcal{O}_{\boldsymbol{P}^{n-1}}(1)^{\oplus 2}$. We infer that there exists an ample line bundle $A$ on $X$ such that $\left(D^{\prime}, A_{D^{\prime}}\right) \cong\left(\boldsymbol{P}^{n-1}, \mathcal{O}(1)\right)$ and $\mathscr{E}_{D^{\prime}} \cong \mathcal{O}_{\boldsymbol{P}^{n-1}}(1)^{\oplus 2}$ for every fiber $D^{\prime}$ of $\varphi$ by an argument similar to $[\mathrm{F} 2,(3.3)]$. Then we get $(X, \mathscr{E}) \cong\left(\boldsymbol{P}_{W}(\mathscr{F}), H(\mathscr{F}) \otimes \varphi^{*} \mathscr{G}\right)$ for the vector bundles $\mathscr{F}:=\varphi_{*} \mathcal{O}_{X}(A)$ and $\mathscr{G}:=\varphi_{*}\left(\mathscr{E} \otimes \mathcal{O}_{X}(-A)\right)$ on $W$.

In Case (e), we have $n=3, r=2$ and $f: P \rightarrow Y$ is a simple blow-up that is a component of the reduction morphism: $(P, H) \rightarrow\left(\boldsymbol{P}^{4}, \mathcal{O}(2)\right)$. For the exceptional divisor $E \cong \boldsymbol{P}^{3}$ of $f$, we see that $\left.p\right|_{E}: \boldsymbol{P}^{3} \rightarrow X$ is finite surjective. Hence $X \cong \boldsymbol{P}^{3}$ and then $\rho(Y)=\rho(X)=1$. Thus $f$ is a simple blow-up of $\left(\boldsymbol{P}^{4}, \mathcal{O}(2)\right)$; $Y \cong \boldsymbol{P}^{4}, H=f^{*} \mathcal{O}_{\boldsymbol{P}^{4}}(2)-E$ and $K_{P}=f^{*} \mathcal{O}_{\boldsymbol{P}^{4}}(-5)+3 E$. For every fiber $Z \cong \boldsymbol{P}^{1}$ of $p$, we have $H Z=1$ and $K_{P} \cdot Z=-2$. It follows that $E Z=1$, hence $E$ is a section of $p$. Since $\left(\left.p\right|_{E}\right)^{*}\left(K_{X}+\operatorname{det} \mathscr{E}\right)=\left.\left(K_{P}+2 H\right)\right|_{E}=\mathcal{O}_{P^{3}}(-1)$, we get $\operatorname{det} \mathscr{E}=\mathcal{O}_{\boldsymbol{P}^{3}}(3)$. Then we have $\left.\mathscr{E}\right|_{l} \cong \mathcal{O}_{\boldsymbol{P}^{1}}(1) \oplus \mathcal{O}_{\boldsymbol{P}^{1}}(2)$ for every line $l \cong \boldsymbol{P}^{1}$ in $X \cong \boldsymbol{P}^{3}$, hence $\mathscr{E} \cong \mathcal{O}_{\boldsymbol{P}^{3}}(1) \oplus \mathcal{O}_{\boldsymbol{P}^{3}}(2)$.

Thus we obtain that $(X, \mathscr{E})$ is one of the cases (i), ., (v) of our theorem. Conversely, by (1.2), we find that $g_{2}\left(\boldsymbol{P}_{X}(\mathscr{E}), H(\mathscr{E})\right)=h^{2}\left(\mathcal{O}_{X}\right)=0$ for all the cases (i) $, \ldots,(\mathrm{v})$.

Remark 3.3.1. We see that the case (v) of (3.3) exists for an arbitrary smooth curve $W$. Indeed, let $\mathscr{F}$ (resp. $\mathscr{G}$ ) be an ample and spanned vector bundle of rank $n$ (resp. 2) on $W$. We set $(X, \mathscr{E}):=\left(\boldsymbol{P}_{W}(\mathscr{F}), H(\mathscr{F}) \otimes \varphi^{*} \mathscr{G}\right)$ and $\left(Y, \mathscr{E}^{\prime}\right):=\left(\boldsymbol{P}_{W}(\mathscr{G}), H(\mathscr{G}) \otimes q^{*} \mathscr{F}\right)$, where $\varphi: \boldsymbol{P}_{W}(\mathscr{F}) \rightarrow W$ and $q: \boldsymbol{P}_{W}(\mathscr{G}) \rightarrow W$ are the bundle projections. Then $\mathscr{E}$ is ample and spanned, and $\left(\boldsymbol{P}_{X}(\mathscr{E}), H(\mathscr{E})\right) \cong$ $\left(\boldsymbol{P}_{Y}\left(\mathscr{E}^{\prime}\right), H\left(\mathscr{E}^{\prime}\right)\right)$, hence $g_{2}\left(\boldsymbol{P}_{X}(\mathscr{E}), H(\mathscr{E})\right)=p_{g}(Y)=0$ by (3.1.2).

COROllary 3.4. Let $X$ be a projective manifold of dimension $n \geq 3$ and $\mathscr{E}$ an ample and spanned vector bundle of rank $r \geq 2$ on $X$. Then $g_{2}\left(\boldsymbol{P}_{X}(\mathscr{E}), H(\mathscr{E})\right) \geq 0$, and equality holds if and only if $(X, \mathscr{E})$ is one of the cases of (3.3).

Remark 3.4.1. The classification of pairs $(X, \mathscr{E})$ with $g_{i}\left(\boldsymbol{P}_{X}(\mathscr{E}), H(\mathscr{E})\right)=0$ and $i \geq 3$ is yet to be studied (cf. (1.4.3)).

\section{Appendix. Lanteri's geometric genus}

A. Lanteri $[\mathrm{L}]$ introduced a notion of geometric genus for ample vector bundles.

Definition A.1 ([L,(1.0)]). Let $X$ be a projective manifold of dimension $n$ and $\mathscr{E}$ an ample vector bundle of rank $r<n$ on $X$ with the following property: 
(*) There exists a section $s \in H^{0}(X, \mathscr{E})$ whose zero locus $Z:=(s)_{0}$ is a submanifold of $X$ of the expected dimension $n-r$.

Then the geometric genus $p_{g}(X, \mathscr{E})$ of the pair $(X, \mathscr{E})$ is defined as $p_{g}(Z)$.

Remark A.1.1. When $r=1, \mathscr{E}$ is an ample line bundle $L$ and we see that $p_{g}(X, \mathscr{E})=g_{n-1}(X, L)$. Furthermore, when $\mathscr{E}=L^{\oplus r}$ is the direct sum of $r$ copies of an ample line bundle $L$, we see that $p_{g}(X, \mathscr{E})=g_{n-r}(X, L)$. When $r=n-1$, $p_{g}(X, \mathscr{E})$ is the curve genus of $(X, \mathscr{E})$ (see [LMS]).

Remark A.1.2. When $\mathscr{E}$ is spanned, the property $(*)$ is satisfied by Bertini's theorem. In that case, the definition of $p_{g}(X, \mathscr{E})$ does not depend on the choice of a section $s \in H^{0}(X, \mathscr{E})$ since the property $(*)$ is an open condition.

The following result is corresponding to (1.4), (2.1.2) and (3.1.3).

Proposition A.2 ([L,(1.1)]). Let $X$ be a projective manifold of dimension $n$ and $\mathscr{E}$ an ample vector bundle of rank $r<n$ on $X$ with the property $(*)$ in (A.1). Then $p_{g}(X, \mathscr{E}) \geq h^{n-r}\left(\mathcal{O}_{X}\right)$, and equality holds if $h^{j}\left(K_{X} \otimes \bigwedge^{r-j} \mathscr{E}\right)=0$ for every integer $0 \leq j \leq r-1$.

We have a relation between $p_{g}(X, \mathscr{E})$ and $g_{n-r}(X$, det $\mathscr{E})$ as follows.

THEOREM A.3. Let $X$ be a projective manifold of dimension $n$ and $\mathscr{E}=\bigoplus_{j=1}^{r} L_{j}$ the direct sum of ample and spanned line bundles $L_{j}$ on $X$. Suppose that $1<r<n$. Then $g_{n-r}(X, \operatorname{det} \mathscr{E}) \geq p_{g}(X, \mathscr{E})$, and equality holds if and only if $g_{n-r}(X, \operatorname{det} \mathscr{E})=p_{g}(X, \mathscr{E})=h^{n-r}\left(\mathcal{O}_{X}\right)$.

Proof. Since every $L_{j}$ is ample and spanned, we can take a general member $D_{j}$ of $\left|L_{j}\right|$ such that $Z:=\bigcap_{j=1}^{r} D_{j}$ is a submanifold of $X, \operatorname{dim} Z=n-r$ and $p_{g}(X, \mathscr{E})=h^{0}\left(K_{Z}\right)$. On the other hand, we can take general members $E_{k}$ of $\mid$ det $\mathscr{E} \mid(1 \leq k \leq r)$ such that $Y:=\bigcap_{k=1}^{r} E_{k}$ is a submanifold of $X, \operatorname{dim} Y=$ $n-r$ and $g_{n-r}(X$, det $\mathscr{E})=h^{0}\left(K_{Y}\right)$. For every integer $1 \leq l \leq r$, we set $Z_{l}:=$ $\bigcap_{j=1}^{l} D_{j}$ and $Y_{l}:=\bigcap_{k=1}^{l} E_{k}$. We may assume that $Z_{l}, Y_{l}$ and $Y_{s} \cap Z_{t}(1 \leq s$, $t \in \boldsymbol{Z} ; s+t=l)$ are submanifolds of $X$ of dimension $n-l$. Then we have $h^{0}\left(K_{Y_{1}}\right) \geq h^{0}\left(K_{Z_{1}}\right)$ because

$$
h^{0}\left(K_{Z_{1}}\right)=h^{0}\left(K_{D_{1}}\right)=h^{0}\left(K_{X}+L_{1}\right)-h^{0}\left(K_{X}\right)+h^{1}\left(K_{X}\right)
$$

and

$$
h^{0}\left(K_{Y_{1}}\right)=h^{0}\left(K_{E_{1}}\right)=h^{0}\left(K_{X}+L_{1}+\cdots+L_{r}\right)-h^{0}\left(K_{X}\right)+h^{1}\left(K_{X}\right) .
$$

We note that if $h^{0}\left(K_{Y_{1}}\right)=h^{0}\left(K_{Z_{1}}\right)$, then $h^{0}\left(K_{X}+\operatorname{det} \mathscr{E}\right)=0$, otherwise we get $h^{0}\left(K_{X}+L_{1}\right)=h^{0}\left(K_{X}+L_{1}+\cdots+L_{r}\right)>0$ and

$$
h^{0}\left(K_{X}+L_{1}+\cdots+L_{r}\right) \geq h^{0}\left(K_{X}+L_{1}\right)+h^{0}\left(L_{2}+\cdots+L_{r}\right)-1>h^{0}\left(K_{X}+L_{1}\right),
$$

which is a contradiction. Similarly we find that 


$$
\begin{gathered}
h^{0}\left(K_{Y_{2}}\right) \geq h^{0}\left(K_{Y_{1} \cap Z_{1}}\right) \geq h^{0}\left(K_{Z_{2}}\right), \\
h^{0}\left(K_{Y_{3}}\right) \geq h^{0}\left(K_{Y_{2} \cap Z_{1}}\right) \geq h^{0}\left(K_{Y_{1} \cap Z_{2}}\right) \geq h^{0}\left(K_{Z_{3}}\right), \\
\cdots, \\
h^{0}\left(K_{Y_{r}}\right) \geq h^{0}\left(K_{Y_{r-1} \cap Z_{1}}\right) \geq \cdots \geq h^{0}\left(K_{Y_{1} \cap Z_{r-1}}\right) \geq h^{0}\left(K_{Z_{r}}\right) .
\end{gathered}
$$

Hence we get $g_{n-r}(X$, det $\mathscr{E})=h^{0}\left(K_{Y_{r}}\right) \geq h^{0}\left(K_{Z_{r}}\right)=p_{g}(X, \mathscr{E})$. Suppose that $g_{n-r}(X, \operatorname{det} \mathscr{E})=p_{g}(X, \mathscr{E})$. Then we infer that $h^{0}\left(K_{Y_{r-1}}+\operatorname{det} \mathscr{E}_{Y_{r-1}}\right)=0$ similarly as above. From an exact sequence

$$
0 \rightarrow \mathcal{O}\left(K_{Y_{r-1}}\right) \rightarrow \mathcal{O}\left(K_{Y_{r-1}}+\operatorname{det} \mathscr{E}_{Y_{r-1}}\right) \rightarrow \mathcal{O}\left(K_{Y_{r}}\right) \rightarrow 0,
$$

we get $h^{0}\left(K_{Y_{r}}\right)=h^{1}\left(K_{Y_{r-1}}\right)$. Since $h^{1}\left(K_{Y_{r-1}}\right)=h^{n-r}\left(\mathcal{O}_{Y_{r-1}}\right)=h^{n-r}\left(\mathcal{O}_{X}\right)$, we see that $g_{n-r}(X, \operatorname{det} \mathscr{E})=h^{n-r}\left(\mathcal{O}_{X}\right)$.

Remark A.3.1. Suppose that $r=n-1$. In general, $g_{1}(X, \operatorname{det} \mathscr{E})>p_{g}(X, \mathscr{E})$ for all ample vector bundles $\mathscr{E}$ on $X$ because

$$
\begin{aligned}
2 g_{1}(X, \operatorname{det} \mathscr{E})-2 & =\left(K_{X}+(n-1) c_{1}(\mathscr{E})\right) c_{1}(\mathscr{E})^{n-1} \\
& >\left(K_{X}+c_{1}(\mathscr{E})\right) c_{n-1}(\mathscr{E}) \\
& =2 p_{g}(X, \mathscr{E})-2 .
\end{aligned}
$$

We note that the curve genus $p_{q}(X, \mathscr{E})$ is defined for all generalized polarized manifolds $(X, \mathscr{E})$ by the last equality above. Pairs $(X, \mathscr{E})$ with $p_{g}(X, \mathscr{E})=h^{1}\left(\mathcal{O}_{X}\right)$ have been classified by [LMS, Theorem] when $\mathscr{E}$ is ample and spanned.

Remark A.3.2. Suppose that $r=n-2$ in (A.3). Because of (2.3), we see that $g_{2}(X$, det $\mathscr{E})>p_{g}(X, \mathscr{E})$ except for $\left(\boldsymbol{P}^{4}, \mathcal{O}(1)^{\oplus 2}\right)$. Moreover, the difference is not necessarily small. When $(X, \mathscr{E})=\left(\boldsymbol{P}^{2} \times \boldsymbol{P}^{2}, \mathcal{O}(1,1)^{\oplus 2}\right)$, for example, we find that $p_{g}(X, \mathscr{E})=0$ and $g_{2}(X, \operatorname{det} \mathscr{E})=9$ by simple computations. Pairs $(X, \mathscr{E})$ with $p_{g}(X, \mathscr{E})=h^{2}\left(\mathcal{O}_{X}\right)$ have been classified by $[\mathrm{L},(3.2) \&(3.3)]$.

Acknowledgment. The author would like to express his gratitude to Professor Y. Fukuma for sending the author interesting preprints including [Fk1,2], also for valuable comments on a draft of the present paper. He also thanks the referee whose corrections and comments made this paper clearer.

\section{REFERENCES}

[BS] M. C. Beltrametti and A. J. Sommese, The Adjunction Theory of Complex Projective Varieties, Expositions in Math., vol. 16, de Gruyter, 1995.

[BiLl] A. Biancofiore, A. Lanteri and E. L. Livorni, Ample and spanned vector bundles of sectional genera three, Math. Ann. 291 (1991), 87-101.

[F0] T. Fusta, Classification Theories of Polarized Varieties, London Math. Soc. Lecture Note Ser., vol. 155, Cambridge University Press, 1990. 
[F1] T. Fujita, Ample vector bundles of small $c_{1}$-sectional genera, J. Math. Kyoto Univ. 29 (1989), 1-16.

[F2] T. FuJitA, On adjoint bundles of ample vector bundles, in Complex Algebraic Varieties (Bayreuth, 1990), Lecture Notes in Math., vol. 1507, Springer, 1992, pp. 105-112.

[Fk1] Y. Fukuma, On the sectional geometric genus of quasi-polarized varieties, I, to appear in Comm. Alg.

[Fk2] Y. FukUma, Projective manifolds with the third sectional geometric genus zero, preprint.

[FkI] Y. Fukuma AND H. Ishinara, A generalization of curve genus for ample vector bundles, II, Pacific J. Math. 193 (2000), 307-326.

[L] A. LANTERI, Geometric genera for ample vector bundles with regular sections, Rev. Mat. Complut. 13 (2000), 33-48.

[LMS] A. LANTERI, H. Maeda AND A. J. Sommese, Ample and spanned vector bundles of minimal curve genus, Arch. Math. (Basel) 66 (1996), 141-149.

[Lz] R. LAZARSFELD, Some applications of the theory of positive vector bundles, in Complete Intersections (Acireale, 1983), Lecture Notes in Math., vol. 1092, Springer, 1984, pp. 29-61.

[OSS] C. Okonek, M. Schneider and H. Spindler, Vector Bundles on Complex Projective Spaces, Progr. Math., vol. 3, Birkhäuser, 1980.

[PS] K. H. Paranjape and V. SRinivas, Self maps of homogeneous spaces, Invent. Math. 98 (1989), 425-444.

[Sa] E. SATo, Varieties which have two projective space bundle structures, J. Math. Kyoto Univ. 25 (1985), 445-457.

[YZ] Y. G. YE AND Q. ZhANG, On ample vector bundles whose adjunction bundles are not numerically effective, Duke Math. J. 60 (1990), 671-687.

FACULTY OF SCIENCE

YoKohama City UnIVERSITY

KanAZAWA-KU, YOKOHAMa 236-0027

JAPAN 